\title{
Epidemiology of Trachoma in the West Bank and Gaza Strip
}

\author{
L. C. CHUMBLEY and I. M. THOMSON \\ Jerusalem
}

\begin{abstract}
Summary
A detailed study was made of the prevalence and associations of trachoma in a prospective, randomised population based study of 9058 Palestinian Arabs living in the West Bank and Gaza Strip, and in a prospective study of 1,000 consecutive unselected ophthalmic outpatients at St John Ophthalmic Hospital, Jerusalem. Trachoma was found to be widely distributed throughout the two regions. This chiefly occurred as a mild, self limited disease, interspersed with discontinuous pockets of blinding disease. The highest prevalence was found in the Hebron and Jericho districts. Sub-populations at increased relative risk included females, older age groups, rural dwellers, patients with pre-disabling or disabling lesions, and patients with moderate to severe active trachoma. An increased recovery of bacteria by culture of the lids and conjunctiva of patients with moderate to severe trachoma was noted. Prevalences of lacrimal disease, dacryocystitis, and acute bacterial ulcer were significantly greater among outpatients with trachoma than among those without trachoma. No difference in prevalence of either mucopurulent conjunctivitis or herpetic dendritic keratitis was demonstrated between patients with and without trachoma. An increased prevalence of trachoma was found in communities without a continuous year-round supply of running water compared with communities with this facility.
\end{abstract}

The West Bank and Gaza Strip offer special opportunities to study the edpidemiology of trachoma. There has been long-standing high endemicity of blinding trachoma in these two regions. Periodic surveys and treatment projects have been performed in the two regions. Several of the latter are models of public health campaigns against trachoma. A further unique factor is the continuous provision of specialist eye care to the area by the same institution, St John Ophthalmic Hospital of Jerusalem, for over a century. It was felt that an updated report on the epidemiology of trachoma in the West Bank and Gaza Strip would be of considerable interest.

\section{Historical Summary}

Essentially, no eye care was available in the Ottoman Empire's province of Palestine before the St John Ophthalmic Hospital was built in Jerusalem in 1882. Before the discovery that sulfonamides were effective against trachoma (1939), the treatment given here was the classical one: silver nitrate, protargol, argyrol, copper.

In 1934 MacCallan wrote that "Palestine has the distinction of having a greater percentage of blind persons among her general population than any other country in the world $(8.4 \%)$...... Practically all the indigenous inhabitants of Palestine have trachoma". ${ }^{1}$ 
Sidky and Freyche ${ }^{2}$ quoted the following prevalence figures for trachoma in Palestine in 1939:

$37 \%$ for urban schoolchildren

$58 \%$ for rural school children, and

$70 \%$ in ophthalmic clinics.

They quoted the prevalence for trachoma in Jordan in 1948 as:

$70 \%$ for the general population, and

$27 \%$ (new cases) in public health clinics.

From 1950 to 1952 , Bietti ${ }^{3}$ conducted prevalence surveys and mass treatment projects under the auspices of the World Health Organisation and in cooperation with St John Ophthalmic Hospital (WHO), and the United Nations Works Relief Agency, among the Arab refugee population. He found that the prevalence rate of trachoma (all stages) among the Arab refugee population varied from $50 \%$ to $90 \%$ according to zone.

In 1955, a WHO survey by $\mathrm{Abdin}^{4}$ gave a prevalence of $72 \%$ in the population for all stages of trachoma in Jordan as a whole. In 1958 Gilkes, 5 in his studies on trachoma in Jordan, found figures of a similar order. As Gilkes studies were not designed to estimate prevalences, he could not extra-polate his figures to the population as a whole.

Winkler in $1963^{6}$ reported a prevalence for trachoma of $48 \%$ ( $31 \%$ active) in the urban, and $64 \%$ (44\% active) in the rural population of the Hebron district, Jordan. This was followed by a mass trachoma treatment campaign in the Hebron district from 1963 to 1965 under the auspices of World Health Organisation and in cooperation with St John Ophthalmic Hospital and the Jordan Ministry of Health, among the school-age population. In 1984, Thomson and Chumbley ${ }^{7}$ found a prevalence for trachoma of $28 \%$ (20\% active) among the general population of the West Bank and Gaza Strip. This was followed by mass trachoma treatment campaigns in Hebron and Jericho districts from 1983 to 1985 under the auspices of St John Ophthalmic Hospital Outreach Programmes and in co-operation with the Institute of Ophthalmology, London.

\section{Methods}

The figures quoted in this report derive from two main sources:

(1) A randomised, prospective, populationbased survey of the prevalence of eye disease in the West Bank and Gaza Strip, performed during 1982 and 1983. Primary sampling units consisted of East Jerusalem, West Bank districts (Jordanian boundaries), and the Gaza Strip considered as a whole. Second stage sampling units consisted of all cities for the urban stratum with number of clusters allotted according to size measures. Camp and rural strata were selected by enumeration and use of a random number table for randomisation. Specific clusters were chosen by a technique of map grid co-ordinate randomisation. (The sample design and some of the results of this survey have been published elsewhere. $^{7}$

(2) A prospective survey of 1,000 consecutive unselected ophthalmic outpatients at St John Ophthalmic Hospital, Jerusalem was carried out during 1982. Detailed eye examinations were performed for every patient, including best corrected visual acuity, Haag Streit slit lamp biomicroscopy, and indirect ophthalmoscopy. Goldmann tonometry was done for patients over the age of 40 years. All upper eyelids of every patient were everted and studied. A detailed proforma was completed for every patient using the classification for parameters of trachoma recommended by the World Health Organisation. Stages of trachoma (MacCallan classification) and computed grades of intensity of inflammation and disabling lesions of trachoma were classified according to currently accepted criteria. ${ }^{8,9}$

Although not recorded in this report, the following sources were also reviewed:

(1) Study of detailed records of mass trachoma treatment campaigns conducted by St John Ophthalmic Hospital, Jerusalem, in co-operation with the Institute of Ophthalmology, London, from 1983 to 1985.

(2) Study of detailed records of mobile eye 
clinics run by St John Ophthalmic Hospi- of the West Bank and Gaza Strip. Fig. 2 tal, Jerusalem, from 1981 to the present.

shows the breakdown of trachoma by stages,

(3) Study of annual hospital statistics at St

John Ophthalmic Hospital, Jerusalem.

\section{Results}

Fig. 1 shows the distribution of trachoma (acin the general population of the West Bank and Gaza Strip.

The sex distribution for persons with trachoma (all stages) in the general population was $43 \%$ male, and $57 \%$ female. The tive Stages I - III) in the general population distribution of stages of trachoma (Mac Cal-

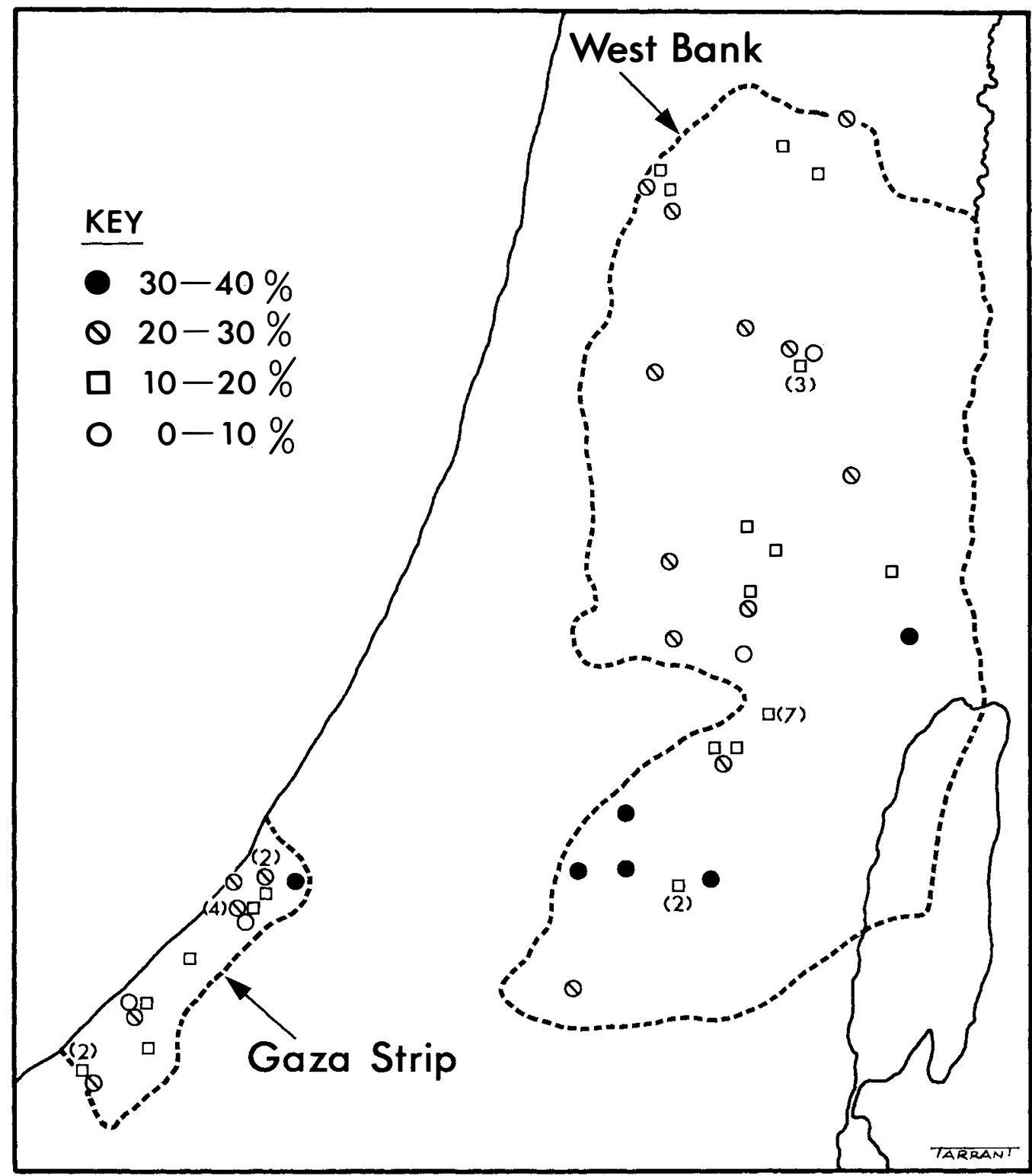

Fig 1. Distribution of active trachoma (Mac Callan Stages I-III) in the West Bank and Gaza Strip. 0 least severe $\emptyset$ - most severe 
lan classification) in the general population was the same for both sexes.

The age distribution varied according to Stage. The active stages were much more prevalent in younger patients, being highest in children younger than 5 years of age, followed by children 5 - 9 years of age. Lower

\section{STAGE TRACHOMA}

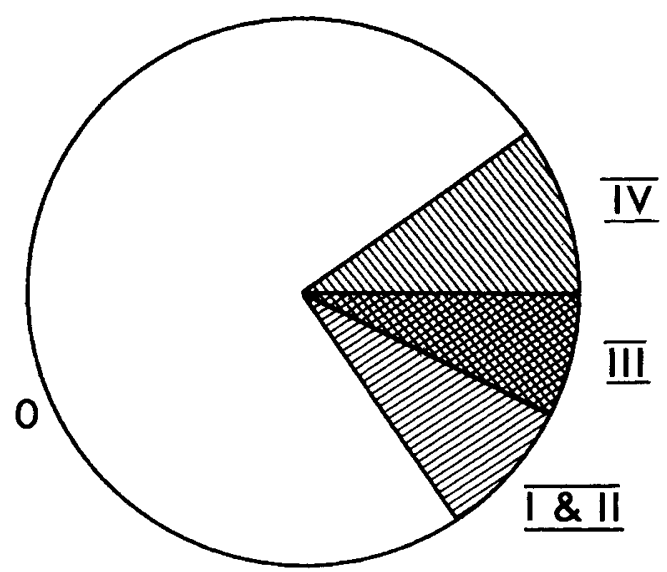

Fig 2. Prevalences of Stages of trachoma (MacCallan classification) in general population of the West Bank and Gaza Strip. $\mathbf{N}=9058$.

\section{GRADE TRACHOMA}

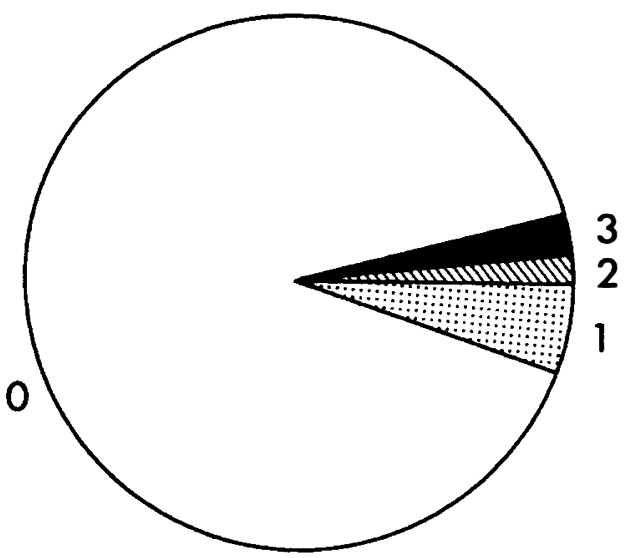

Fig 3. Prevalences of Grades of trachoma in ophthalmic outpatients at St John Ophthalmic Hospital, Jerusalem.

$(0=$ nil or trivial, $1=$ mild, $2=$ moderate, $3=$ severe) $\mathrm{N}=1,000$. prevalence of active trachoma occurred with increasing age. The prevalence of cicatricial and inactive stages of the disease rose with increasing age, though all age groups were represented, including pre-school children.

Fig. 3 shows the prevalence of grades of intensity of trachoma among ophthalmic outpatients at St John Ophthalmic Hospital. In several villages where treatment campaigns were carried out and accurate figures were available, the prevalence of moderate to severe (Grades 2 and 3) trachoma was of the order of $5 \%$. The distribution of grades of intensity of inflammation was the same for both males and females for both ophthalmic out-patients and for several selected villages for whom the entire population was examined. We do not have a comparable figure for the general population as a whole.

The prevalence of trichiasis in the general population was $0.7 \%$, rising to $9.5 \%$ among ophthalmic outpatients.

A series of patients with moderate to severe intensity of trachoma had lid and conjunctival swabbing performed for bacterial culture. Of the lid cultures, $5 \%$ of the patients were negative and $95 \%$ were positive. Of the conjunctival cultures, $23 \%$ of the patients were negative and $77 \%$ were positive. Principal organisms cultured were Staph aureus, Staph albus, and alpha streptococcus.

The distribution of potentially disabling (distortion of the eyelids due to conjunctival scarring, and trichiasis and/or entropion) and disabling (severe central corneal scarring with gross visual loss) trachomatous lesions were the same between males and females among ophthalmic outpatients. Overall, potentially disabling lesions were present in $12 \%$, and disabling trachomatous lesions in $8 \%$, of the ophthalmic outpatients.

Fig. 4 shows the prevalence of binocular blindness due to trachoma in the general population according to rural, urban, or camp stratification. Fig. 5 shows the prevalence of dacryocystitis among ophthalmic outpatients with and without trachoma.

The prevalence of lacrimal disèase of any kind was greater among ophthalmic outpatients with trachoma $(5.4 \%)$ than among patients without trachoma $(2.9 \%)(\mathrm{P}<0.05)$.

Prevalence of lacrimal disease was greater 
with the following features of trachoma: increasing cicatrisation, presence of Herbert's pits, increasing limbal and corneal vascularisation.

Fig. 6 shows the prevalence of acute bacterial ulcers among ophthalmic outpatients with and without trachoma.

Fig. 7 shows the prevalence of trachoma in the general population in villages with and without a continuous year round water supply.

Presence of trachoma did not correlate with presence of mucopurulent conjunctivitis for both the general population and the ophthalmic outpatients. There was no difference in prevalence of herpetic dendritic keratitis among ophthalmic outpatients with and without trachoma.

\section{Discussion}

Before reviewing the findings, one may pose four main questions. First, what are the particular epidemiologic characteristics of trachoma in the West Bank and Gaza Strip now, and what might account for the patterns found? Secondly, what direction might the disease patterns appear to be taking historically in the dimension of time? Thirdly, what associations might shed light on the multifactorial pathogenesis of blinding trachoma and blinding ophthalmia? Fourthly, which populations appear to be at increased risk for blinding complications of this disease?

The epidemiologic characteristics of trachoma found in the West Bank and Gaza Strip may be summarised as follows. Trachoma was found to be widely distributed throughout these two regions. There was considerable variation in prevalence from community to community. The distribution pattern was not regular nor of a type where graded isobars could be used to describe its appearance. The distribution was found to be patchy, interspersed and discontinuous. The geographic districts of greatest prevalence, that is, the Hebron and Jericho districts, have proportionately less rainfall than the other districts. The Jordan Valley, which contains the Jericho district, is $-345 \mathrm{~m}$ elevation, hot and dry during summer and mild and dry during winter. The Hebron hills are the highest

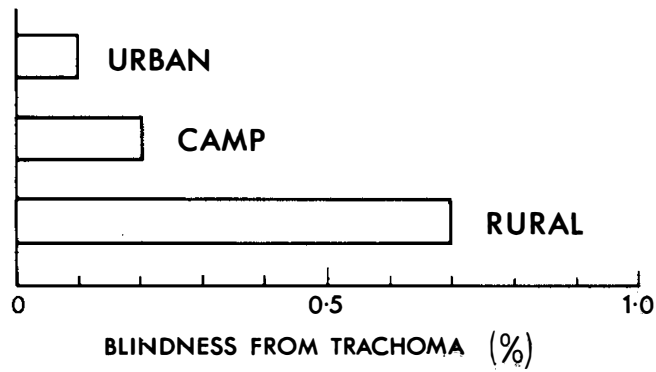

Fig 4. Bar chart showing prevalence of binocular blindness from trachoma in general population of the West Bank and Gaza Strip, according to urban, camp or rural strata. Differences shown are statistically significant. $N=9058$.

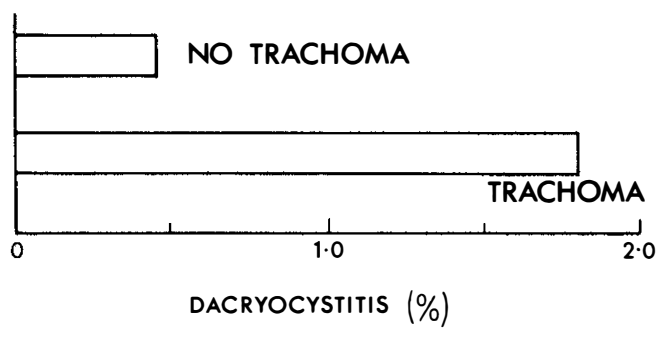

Fig 5. Bar chart showing prevalence of dacryocystitis among ophthalmic outpatients at St John Ophthalmic Hospital, Jerusalem, with and without trachoma $(P=0.01)$. N 1,000.

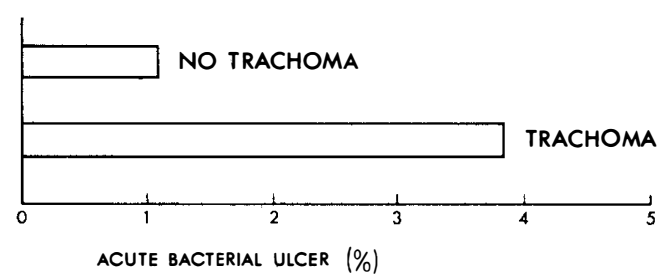

Fig 6. Bar chart showing prevalence of acute bacterial ulcer among ophthalmic outpatients at $S t$ John Ophthalmic Hospital, Jerusalem, with and without trachoma. $(P=0.01)$. N 1,000.

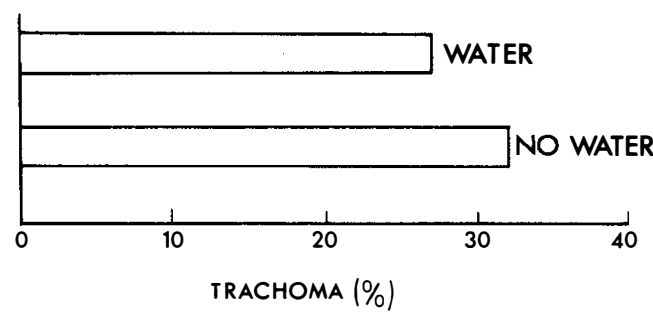

Fig 7. Bar chart showing prevalence (all stages) in patients from communities with and without continuous year-round supply of running water $(P=0.001)$.N 9,058. 
part of the West Bank and Gaza Strip, and have somewhat less rainfall than the other upland parts of the West Bank.

Many factors are reflected in the prevalence of trachoma in a given community. The previous or earlier degree of endemicity in a community is part of its health heritage. Acting on this are social and hygiene practices, sanitation, fly populations and water supply. The availability of medical care and of pharmacies also have an influence. The effects of specific campaigns against trachoma will be reflected as well.

Trachoma in the West Bank and Gaza Strip was found to be mainly a mild, selflimited condition. Against this back-ground of widespread mild disease pockets of blinding trachoma were found. The two milieux can interact possibly to perpetuate one another.

Prevalence of blindness resulting from trachoma shows a marked difference between rural dwellers $(0.7 \%)$ as opposed to camp $(0.2 \%)$ and urban $(0.1 \%)$ dwellers. The timeless rhythm of village life in many communities, coupled with conservative tradition, perpetuates social, hygienic, and sanitation practices and resistance to change. Of special interest was the reduced prevalence of blinding trachoma among camp dwellers. Refugees or displaced persons, most of whom are of rural background, would have been expected to have poorer health status than non-refugees. That theirs was better than non-refugee villagers reflects, in our opinion, the favourable results of intensive trachoma campaigns in the 1950s among the refugee population. Higher grades of inflammation were found in this sub-population however, so an attitude of false assurance must be guarded against. Nevertheless, the above-mentioned campaigns do appear to have been effective in reducing the blinding effects of trachoma for several generations of refugees. ${ }^{10}$ Urban dwellers had the lowest prevalence of blinding trachoma. This may reflect improved economic status, increased awareness of and level of exposure to health and education and new information and better access to water and sanitation infrastructure and to medical and pharmacy services.
Trachoma in the West Bank and Gaza Strip affected females more than males. This has been found in previous studies elsewhere and has generally been explained as follows. Mothers, grandmothers and small children perpetuate the cycle of reinfection in the home. Refuse and food lying about attracts flies, which aid transmission between the generations. The elderly are at increased risk to develop blinding complications, since these may occur from progressive cicatrisation abetted by recurrent infections over the years. As expected, active disease was more prevalent in the younger age groups while inactive disease was more prevalent in the older age groups.

Trachoma is known to be distributed widely in many hot arid countries. We found that residents of villages which did not have a regular year-round water supply had a higher prevalence of trachoma. Though the difference was not large in absolute terms, it was found to be statistically significant. We are not aware that this has been shown before and feel that improved water supply may promote better eye health in these communites. As many different factors are involved in assessing water supply it is important to rule out possible confounding variables such as economic status of villages, education of villages and other climatic factors which may influence the prevalence of trachoma. In historic terms, at least over the past half century or more for which data are available, it would appear that the trend in this region has been one of progressive improvement. This reflects sustained public health efforts, coupled with the progressively increased technological complexity of the organisation of society in general, such as communications, transportation and infra-structure. Against the security of a favourable trend, it must be noted that for a villager in these areas the prevalence of blindness from a single preventable cause, trachoma, is 3.5 - 7 times that found in the United Kingdom or United States of America from all causes of blindness put together. ${ }^{7}$ Much work remains to be done in the struggle against blinding trachoma.

This study showed some interesting associtions between trachoma and several other 
ocular conditions which may shed light on the multifactorial pathogenesis of blinding trachoma and blinding ophthalmia.

We found a statistically increased prevalence of dacryocystitis and of lacrimal disease in general in patients with trachoma. Furthermore, the presence of lacrimal disorders was greater with increased degrees of the following features of trachoma: increasing cicatrisation, presence of Herbert's pits, and increasing limbal and corneal vascularisation.

Tabbara and Bobb ${ }^{11}$ reported lacrimal complications in a group of Saudi Arabian patients with severe inactive trachoma. These included dry eye syndrome, punctal phimosis, punctal occlusion, canalicular occlusion, nasolacrimal-duct obstruction, dacryocystitis, dacryocystocoele and dacryocutaneous fistula. They felt that trachoma may cause chronic inflammation of the lacrimal passages, which may lead to cicatrisation of any site in the lacrimal system. They postulated that the high frequency of dacryocystitis is due to the high frequency of nasolacrimal-duct obstruction in patients with late trachoma. Bacteria were recovered in cultures from the eyes of all their patients with dacryocystitis. These findings were confirmed and it was postulated that lacrimal obstruction may possibly add another insult to the trachomatous eye in the multifactorial environment of increased vulnerability.

An increased prevalence of acute bacterial ulcers was found among ophthalmic outpatients with trachoma than among those without trachoma, an association which seems to have been overlooked. Of interest is the fact that Thomson and Chumbley ${ }^{7}$ found that corneal leukoma was the third most prevalent cause of blindness in the West Bank and Gaza Strip, after cataract and trachoma. It is possible that some of the cases of corneal leukoma began as acute bacterial ulcers and that some may have occurred in eyes compromised by the trachomatous process. These may possibly include: micro-trauma by inturning lashes in patients with trachomatous trichiasis, or by concretions in patients with trachomatous tarsal degeneration, or effects of an abnormal tear film or ocular surface abnormality resulting from fibrous replacement of goblet cells and damage to
Meibomian glands or to deficient aqueous lacrimal secretion.

An increased prevalence of mucopurulent conjunctivitis was not demonstrable in patients with trachoma at the time of year in which the survey was done (November to April). The association between trachoma and mucopurulent conjunctivitis has been found to be a seasonal one in other countries e.g. Morocco, Tunisia, and there may well be an association at another time of year when epidemic conjunctivitis is present. Patients with moderate or severe trachoma were found to have an increased number of bacteria recovered in cultures from the conjunctival sac.

No difference in the prevalence of herpes simplex keratitis among patients with and without trachoma was found in this study.

Jones, Darougar, Mohsenine, and Poirier $^{12}$ have written a thought-provoking analysis of communicable ophthalmia in the Middle East. They point to the importance of the inter-relations between acute and chronic ophthalmia, and blinding and non-blinding trachoma. They emphasise the importance of continuing massive pressure of communication of eye infection and the interaction of mucopurulent conjunctivitis with or without corneal ulceration in acute ophthalmia which, occurring in a patient with chronic trachoma, can convert non-blinding trachoma into blinding trachoma.

Combining the risk factors demonstrated in our study, we can state that the subpopulations found at higher risk were females, rural dwellers, elderly patients, patients with potentially disabling or disabling trachomatous lesions, and patients with moderate to severe grades of intensity of active trachoma.

It is hoped that this report, focusing as it does on a particular area at a particular time, will provide some perspective on those contemporary efforts here and elsewhere directed towards reducing the amount of blindness and morbidity engendered worldwide by this major blinding eye disease.

We are grateful to Mrs. J. Thomson and Mr. S. Sharif for their help in carrying out this project, and to the Christoffel Blindenmission, Operation Eyesight Universal, and the Order of St John for financial aid. We are grateful also to Dr. N. Day, 
Biostatistical Department of Imperial Cancer Institute and Cambridge University, who performed the statistical tests.

\section{References}

${ }^{1}$ MacCallan AF: Trachoma in the British Colonial Empire. Its relation to blindness; the existing means of relief; means of prophylaxis. Br J Ophthalmol 1934, 18: 626-45

${ }^{2}$ Sidky MM, Freyche MF: World distribution and prevalence of trachoma in recent years. Epidemiol Vital Statist Rep 1949, II: 11-12: 230280.

${ }^{3}$ Bietti GB: Unpublished working documents WHO/Trachoma/10 and WHO/Trachoma/11 1951.

${ }^{4}$ Abdin G: Unpublished working document WHO/EM/TRACH/7 1956.

${ }^{5}$ Gilkes M: Trachoma in Jordan and Gambia. Trans. Ophthalmol. Soc. UK. 1958; 78: 227-43

${ }^{6}$ Winkler PG: A Morbidity survey on trachoma and other cummunicable eye diseases in the district of Hebron, Jordan, 1960. Bull. Wld. Hlth. Org. 1963; 28: 417-36.

${ }^{7}$ Thomson IM and Chumbley LC: Eye disease in the West Bank and Gaza Strip Br J Ophthalmol 1984, 68: 598-602.

${ }^{8}$ Dawson CR, Jones BR, Tarrizzo ML: Guide to Trachoma Control. World Health Organisation, Geneva, 1981.

${ }^{9}$ Dawson CR, Jones BR, Darougar S: Blinding and non-blinding trachoma: assessment of intensity of upper tarsal inflammatory disease and disabling lesions. Bull. Wld. Hlth. Org. 1975, 52:279-82

${ }^{10}$ Johnson ATG: Unpublished working document WHO/EM/TRACH/15 1959.

${ }^{11}$ Tabbara KF, Bobb AA: Lacrimal system complications in trachoma. Ophthalmology 1980, 298-301.

12 Jones BR, Darougar S, Mohsenine H, Poirier RH: Communicable ophthalmia: the blinding scourge of the Middle East. Br J Ophthalmol 19760: 60:492-8 